\title{
New light absorbing material for grazing angles
}

\author{
A. Yevtushenko ${ }^{1}$, A. von Finck ${ }^{2}$, D. Katsir ${ }^{1}$, A. Duparré ${ }^{2}$ \\ ${ }^{1}$ Acktar Ltd, Kiryat Gat, Israel \\ ${ }^{2}$ Fraunhofer Institute for Applied Optics and Precision Engineering (IOF), Jena, Germany
}

\begin{abstract}
One of the largest challenges for optical systems is eliminating stray light generated by reflections off the walls and other optical elements of the device. Most black coatings are not sufficiently effective at grazing angles. Acktar's black coatings exhibit particularly low residual reflectance and have been implemented in various instruments. The new proposed material exhibits particularly low hemispherical and specular reflectance - especially at grazing angles.
\end{abstract}

\section{INTRODUCTION}

Stray light is a significant issue in almost any optical design, especially in space applications that require high performance, long life stability and maintenance free solutions. The problem takes place mainly in telescopes, star trackers and other satellite sensing devices.

To this date, the best known solution for stray light elimination is by incorporating vanes to the inner walls of baffles and optical housing devices, by allowing a high reduction ratio of specular reflection from the optical components.

In parallel to design solutions, new blackening techniques and materials are being developed to minimize the optical noise in the systems.

The specular reflectance of a surface is defined as the specularly reflected power normalized to the incident power [1], see Fig. 1.

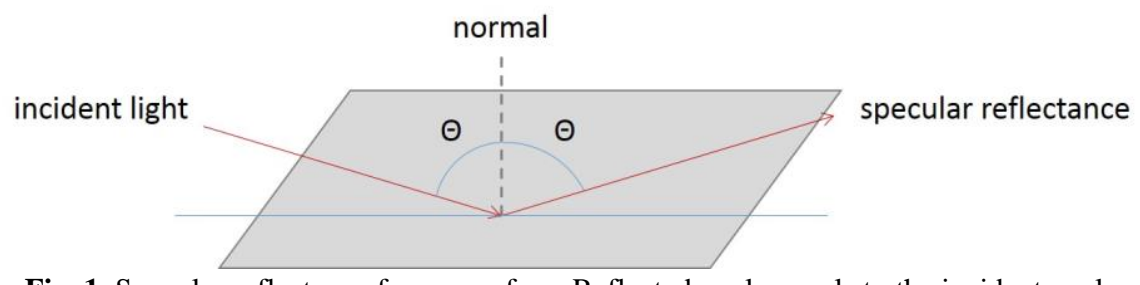

Fig. 1. Specular reflectance from a surface. Reflected angle equals to the incident angle.

\section{VANES SOLUTION FOR STRAY LIGHT REDUCTION}

Vanes are currently the most popular solution for stray light problems. The unwanted light that arrives mainly at grazing angles are blocked by the vanes from reaching the detector, while the direct rays pass through without obstacles.

The designer takes into account the optimum number of vanes, ease of installation, low reflective coatings, etc. (Fig. 2). While these will allow for better performance, it will also add complexity, cost, weight and size to the entire system. 


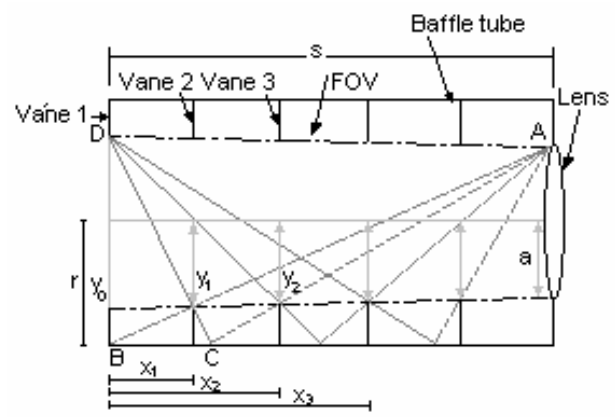

Fig. 2. General design of a baffle tube with vanes, with main dimensions and ray trace of incident light [2].

\section{NEW LIGHT ABSORBING MATERIAL FOR GRAZING ANGLES}

As mentioned above, the vane design's objective is to solve stray light problems in baffles, especially at grazing angles of incidence (when the beam is nearly parallel to the surface). A "grazing angle" is defined as the angle between the beam and the surface (Fig. 3), which is opposite to the definition of an "angle of incidence" (AOI) that is defined as the angle between the beam and the surface normal.

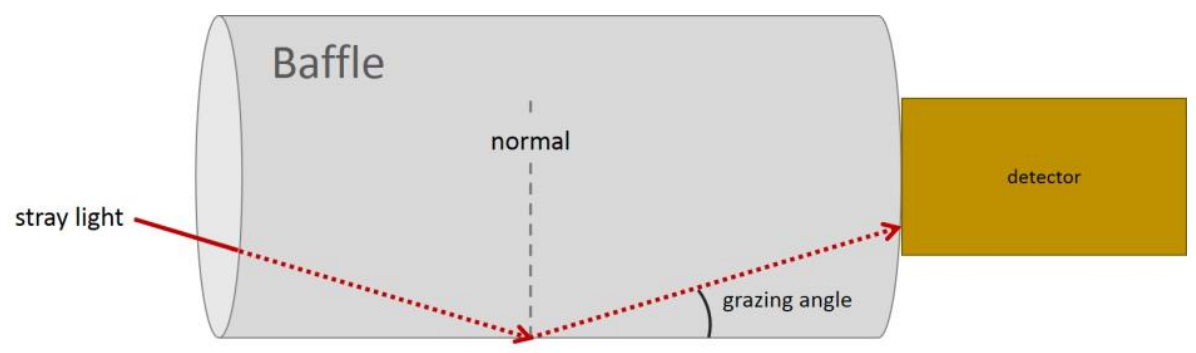

Fig. 3. Grazing angle - the angle between the beam and the surface.

Even though the new light absorbing material offers a solution that focuses on grazing angles, it also provides a solution for stray light from all other angles.

By placing a honeycomb structured surface in the baffle, we create a result similar to that of "many small vanes" as described in our previous paper [1], without the extra weight, size and cost.

\subsection{Cells size selection}

In choosing the optimal ratio between the cell's height and inner size we considered the following:

a. The structure's flexibility- the ability to bend for inserting into the baffle.

b. Minimize the angle of incident at the surfaces that directly reflect onto the detector (Fig. 4 (b)). According to BRDF data, large angles cause higher hemispherical and specular reflectance.

c. Minimize direct reflectance to the detector from sloping edges (Fig. 4 (a)), by reducing the size and number of them.

d. Strength of the structure.

e. Generic design - to minimize calculations and mechanical designing for each unique system. 


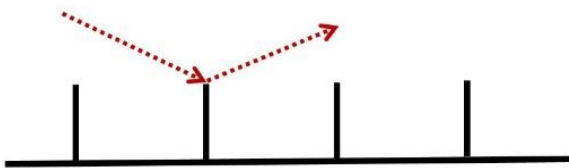

(a)

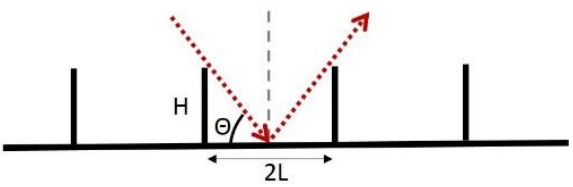

(b)

Fig. 4. Edge mode of the stray light paths - specular reflectance directly to the detector. Path (a) reflected from the sloping edge. Path (b) reflected from the baffle wall.

The cell's height was defined as $2 \mathrm{~mm}$ for maximum flexibility of the material.

To define the inner cell size, we looked at the angle of incident (AOI) of the direct specular reflectance onto the detector off the baffle walls. On a fixed cell height, the maximum AOI will grow with the inner cell size. Taking into account the BRDF behavior of a surface (without considering the surface type) we will see that smaller AOI deliver smaller specular reflectance. Therefore $\Theta_{1}$ (Fig. 5) is a better choice.

On the other hand, too small cells will increase the reflections from the sloping edge (Fig 4. (a) above), as more edges would be added. In this case $\Theta_{3}$ (Fig. 5) is the better choice.

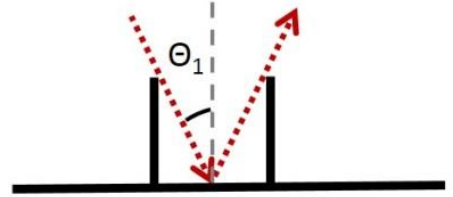

(a)

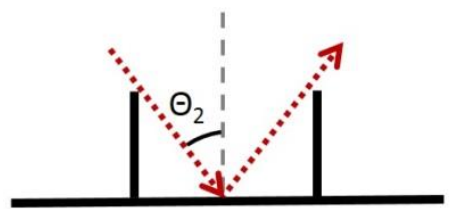

(b)

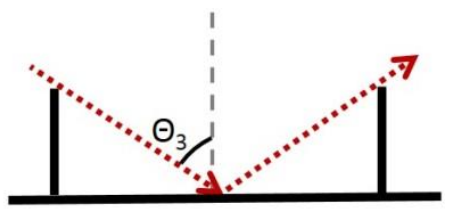

(c)

Fig. 5. Cell size influence on maximum AOI from baffle wall: $\Theta_{1}<\Theta_{2}<\Theta_{3}$.

In defining the optimal inner cell size, we examined BRDF measurements made on a flat surface with Acktar coating. These measurements were performed using an ALBATROSS scatterometer developed with capabilities to measure high-end absorbing surfaces, at Fraunhofer IOF in Jena [3], [4].
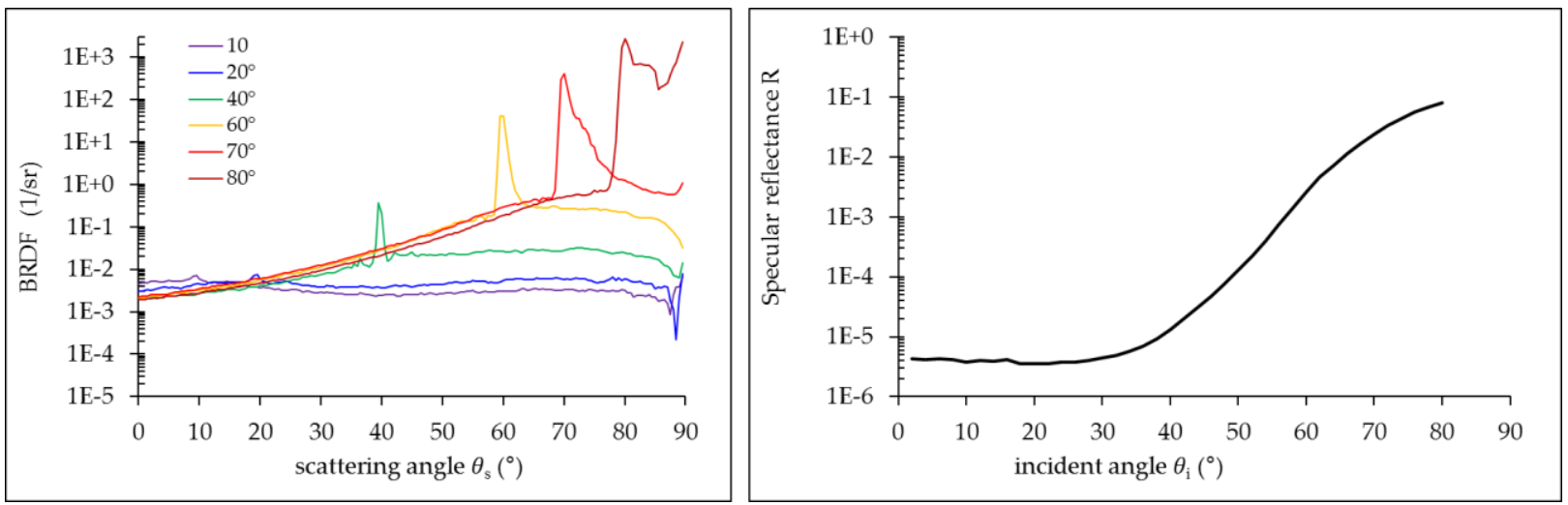

Fig. 6. Left: BRDF measurements at $4.6 \mu \mathrm{m}$ wavelength on Acktar black coating at different incident angles (only positive angles shown). Right: Measured specular reflectance of the same surface as a function of the incident angle

Fig. 6 shows that there are no notable changes in the specular reflectance at angles smaller than $40^{\circ}$, significant changes begin at angles above that. Therefore we defined an inner cell size that would provide a maximum AOI of $40^{\circ}$. The following geometric calculation provided the result. 


$$
\text { inner cell size }=2 \cdot \text { cell height } \cdot \tan \left(40^{\circ}\right)=2 \cdot 2 \mathrm{~mm} \cdot \tan \left(40^{\circ}\right)=3.36 \mathrm{~mm}
$$

Taking into account the standard cells available, the final size was defined as $3.2 \mathrm{~mm}$.

In addition to reducing the number of sloping edges, we wanted to minimize the reflectance from a single sloping edge. Therefore we used a thin (less than $40 \mu \mathrm{m}$ ) foil for the structure. This thickness held the necessary mechanical strength and minimized the surface of impact with stray light. This foil was blackened by Acktar black coating, which was chosen because of its thickness, adding only a few microns.
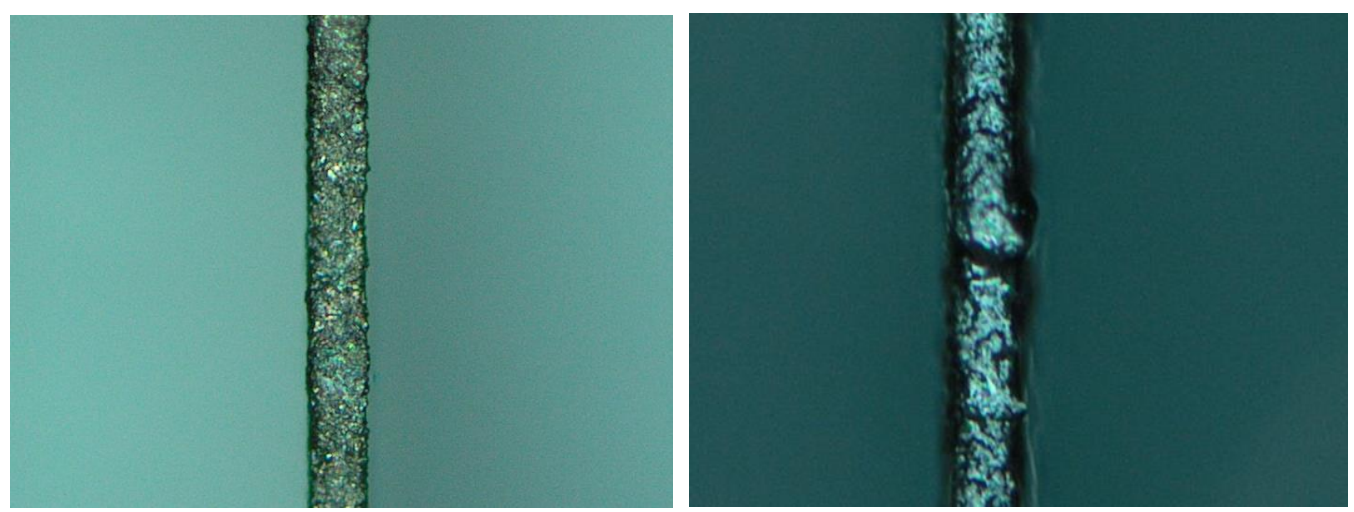

Fig. 7. Cross section of a $38 \mu \mathrm{m}$ aluminum foil coated on both sides, magnification of $\mathrm{x} 100$. Left: Acktar Magic Black coating. Right: Black paint.

\subsection{Black coating for high performance}

While the macrostructure itself is a good start, the black coating is extremely important and should be carefully looked into for different applications. In our investigation we used Acktar Magic Black coating - one of the blackest known to date. Having nearly $1 \%$ of hemispherical reflectance at VIS-NIR spectrum, this coating has many other advantages that make the new developed material very unique:

a. Extremely low reflectance - as mentioned above, having many small vanes means many sloping edges which lead to specular reflectance. This low reflectance coating will reduce most of the unwanted reflectance.

b. Thickness and weight - only a thin uniform layer will minimize the surface area of the sloping edges and reduce the specular reflectance directly to the sensor (coating thickness is about $1 \mu \mathrm{m}$, see Fig. 7), without adding any significant weight.

c. Low outgassing - is a very important property for space, and vacuum applications. Many treatments are developed to reduce the outgassing of the material, however these treatments usually reduce the reflectance advantage. Magic Black has an outgassing of $0.001 \%$ CVCM (Collected Volatile Condensable Materials), $0.2 \%$ RML (Recovered Mass Loss) [5]. Therefore, there is no need for any additional treatments or measures to be taken.

d. Space qualified - Acktar black coatings are qualified for space environment and space applications [6].

e. Resistant to UV - being an inorganic coating, the performance doesn't change. Not even after long exposure to UV light. 


\section{BRDF MEASUREMENTS}

The previous BRDF measurements were performed using the ALBATROSS scatterometer developed at Fraunhofer IOF in Jena [3], [4]. The illumination range of the instrument is between $325 \mathrm{~nm}$ and $10600 \mathrm{~nm}$.

A characterization of this kind of structure (see Fig.8) is especially challenging as [1]:

a. The light scattering distribution is expected to be anisotropic, which would require angle resolved measurements in the full reflection hemisphere. However, hemispherical measurements are especially comprehensive for infrared wavelengths.

b. The lateral dimension of the material's cell size is larger than the illumination spot diameter.

In order to get sample representative measurement data with reasonable effort, BRDF and reflectance was averaged from measurements performed at 5 different measurement positions measured at $4.6 \mu \mathrm{m}$.

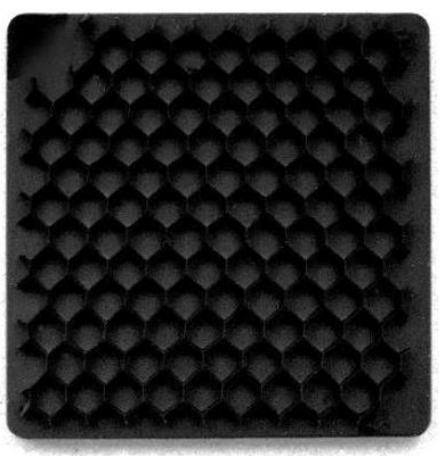

Fig. 8. The proposed new black material (for illustration contrast and brightness of the picture were enhanced)

Assuming the reflectance performance of nearly any material is better at visible wavelengths when compared to infrared, our first evaluation was at $4.6 \mu \mathrm{m}$. The BRDF measurements are shown on Fig. 9.
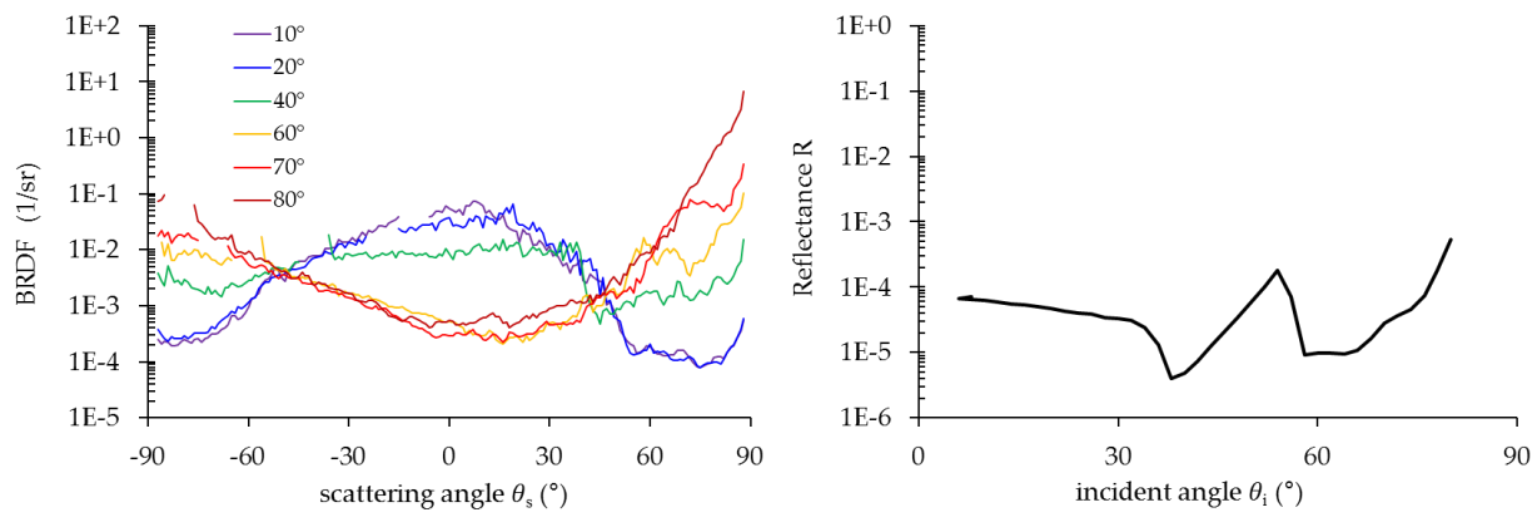

Fig. 9. BRDF (left) and specular reflectance (right) averaged from 5 different measurement positions at wavelength of $4.6 \mu \mathrm{m}$

Additionally, the sample was characterized at VIS spectral ranges, with an illumination wavelength of $640 \mathrm{~nm}$, with a $45^{\circ}$ linear illumination polarization, and a spot diameter of about $3 \mathrm{~mm}$. The results are shown on Fig. 10. This time specular reflectance was not measured as the sample did not show any specular behavior. 


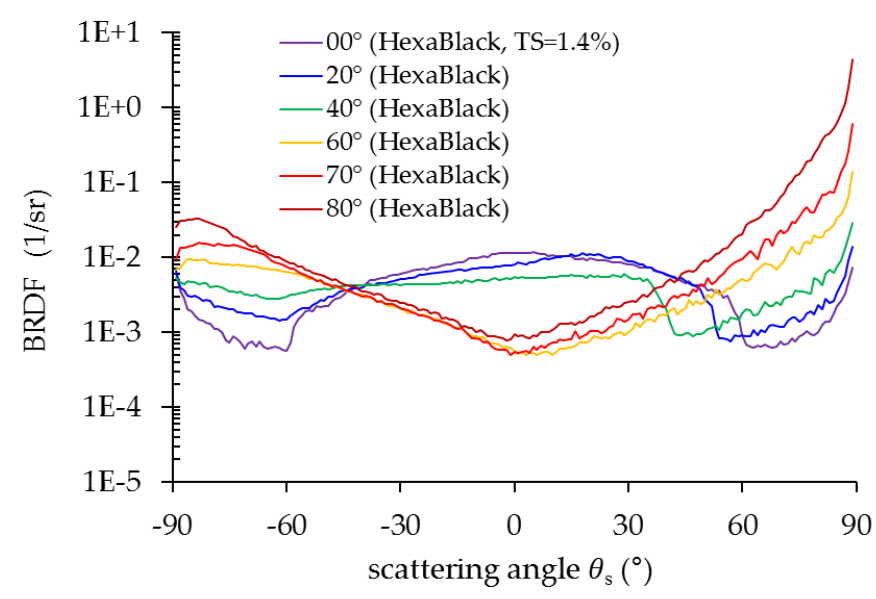

Fig. 10. BRDF averaged from 2 different measurement positions at wavelength of $640 \mathrm{~nm}$.

Analogously to the IR measurements, the BRDF was averaged from the measurements performed on 2 different measurement positions on the same sample.

The performance of the material is similar at both IR and VIS spectral ranges, as shown in Fig. 11. The main difference is at small AOI where IR behavior is better at grazing angles, while the VIS behavior is flatter and more angle independent.
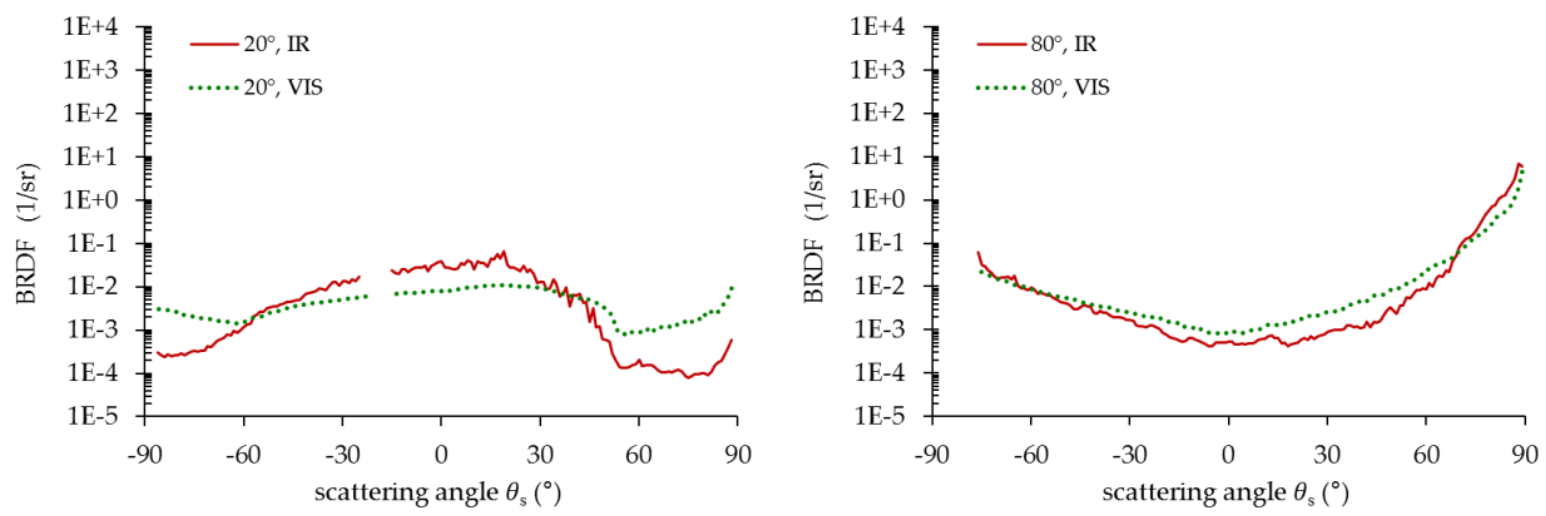

Fig. 11. BRDF comparison of IR and VIS measurements. Left: $A O I=20^{\circ}$. Right: $A O I=80^{\circ}$.

\section{CONCLUSIONS}

In this paper we presented characteristics of an innovative black absorbing material that shows low reflectance at all angles of incidence with an emphasis on grazing angles. You will also find that the material performs almost the same at VIS and IR wavelength ranges. This allows the use of the same material and design for optical systems working at any wavelength from VIS to IR.

Comparing the results of the optical performance of vanes in star trackers and telescopes as described in previous article [1], with the additional results above and all other benefits mentioned we recommend this material as an alternative to common use of vane designs. 
References

[1] A. Yevtushenko, A. v. Finck, D. Katsir, H. Shfaram and A. Duparré, "Vane-free design for star trackers and telescopes," in ICSO, Biarritz, 2016.

[2] C. N. S. Lucimara, É. G. Carvalho, L. F. Santos, F. M. M. Yasuoka, M. A. Stefani and J. C. Castro, "Baffle Design and Analysis of Stray-light in Multispectral Camera of a Brazilian Satellite," Annals of Optics XXIX ENFMC, 2006.

[3] S. Schröder, T. Herffurth, H. Blaschke and A. Duparré, "Angle-resolved scattering: an effective method for characterizing thin-film coatings," Applied Optics, vol. 50, pp. C164-C171, 2011.

[4] S. Schröder, A. von Finck and A. Duparré, "Standardization of light scattering measurements," Advanced Optical Technologies, Vols. 4(5-6), pp. 361-375, 2015.

[5] Acktar Ltd, [Online]. Available: http://www.acktar.com.

[6] Y. M. Salomon, N. A. Sternberg, I. Gouzman, G. Lempert, E. Grossman, D. Katsir, R. Cotostiano and T. Minton, "QUALIFICATION OF ACKTAR VACUUM BLACK ${ }^{\mathrm{TM}}$ COATING FOR SPACE APPLICATION".

[7] J. Y. Plesseria, E. Mazy, J. Defise, P. Rochus, E. Lemmens and D. Vrancken, "Optical and mechanical design of a straylight rejection baffle for COROT". 